\title{
The Behavior of Positive Solutions of a Nonlinear Second-Order Difference Equation
}

\author{
Stevo Stević ${ }^{1}$ and Kenneth S. Berenhaut ${ }^{2}$ \\ ${ }^{1}$ Mathematical Institute of the Serbian Academy of Science, Knez Mihailova 35/I, \\ 11000 Beograd, Serbia \\ ${ }^{2}$ Department of Mathematics, Wake Forest University, Winston-Salem, \\ NC 27109, USA
}

Correspondence should be addressed to Stevo Stević, sstevo@matf.bg.ac.yu

Received 16 August 2007; Accepted 8 December 2007

Recommended by Allan C. Peterson

This paper studies the boundedness, global asymptotic stability, and periodicity of positive solutions of the equation $x_{n}=f\left(x_{n-2}\right) / g\left(x_{n-1}\right), n \in \mathbb{N}_{0}$, where $f, g \in C[(0, \infty),(0, \infty)]$. It is shown that if $f$ and $g$ are nondecreasing, then for every solution of the equation the subsequences $\left\{x_{2 n}\right\}$ and $\left\{x_{2 n-1}\right\}$ are eventually monotone. For the case when $f(x)=\alpha+\beta x$ and $g$ satisfies the conditions $g(0)=1, g$ is nondecreasing, and $x / g(x)$ is increasing, we prove that every prime periodic solution of the equation has period equal to one or two. We also investigate the global periodicity of the equation, showing that if all solutions of the equation are periodic with period three, then $f(x)=c_{1} / x$ and $g(x)=c_{2} x$, for some positive $c_{1}$ and $c_{2}$.

Copyright ( $\odot 2008$ S. Stević and K. S. Berenhaut. This is an open access article distributed under the Creative Commons Attribution License, which permits unrestricted use, distribution, and reproduction in any medium, provided the original work is properly cited.

\section{Introduction}

Recently there has been great interest in the study of nonlinear and rational difference equations (cf. [1-35] and the references therein).

In this paper, we study the boundedness, global asymptotic stability, and periodicity for positive solutions of the equation

$$
x_{n}=\frac{f\left(x_{n-2}\right)}{g\left(x_{n-1}\right)}, \quad n \in \mathbb{N}_{0},
$$

where $f, g \in C[(0, \infty),(0, \infty)]$. 


\section{Asymptotic periodicity of (1.1)}

In this section,we investigate asymptotic periodicity of (1.1). The asymptotic periodicity of some difference equations has been investigated, for example, in the papers $[3-6,10,12,15,20$, $22,23,25-29,31,32,35]$. Our first result is the following theorem regarding eventual monotonicity, which is a natural extension of [24, Theorem 2].

Theorem 2.1. Assume that $f$ and $g$ are nondecreasing functions which map the interval $(0, \infty)$ into itself, and assume that $\left\{x_{n}\right\}$ is a solution of (1.1). Then, the sequences $\left\{x_{2 n}\right\}$ and $\left\{x_{2 n+1}\right\}$ are eventually monotone.

Proof. Suppose that $\left\{x_{n}\right\}$ is a solution to (1.1), and set

$$
r_{n}= \begin{cases}1 & \text { if } \frac{x_{n}}{x_{n-2}} \geq 1 \\ 0 & \text { otherwise }\end{cases}
$$

for $n \geq 0$. Note that it suffices to show that $\left\{r_{2 i}\right\}_{i \geq 0}$ and $\left\{r_{2 i+1}\right\}_{i \geq 0}$ are eventually constant.

From (1.1), we have that

$$
\frac{x_{n}}{x_{n-2}}=\frac{f\left(x_{n-2}\right)}{g\left(x_{n-1}\right)} \frac{g\left(x_{n-3}\right)}{f\left(x_{n-4}\right)}=\frac{f\left(x_{n-2}\right)}{f\left(x_{n-4}\right)} \frac{g\left(x_{n-3}\right)}{g\left(x_{n-1}\right)} .
$$

If $r_{n-2}=1$ and $r_{n-1}=0$, then by (1.1) and the monotonicity of $f$ and $g$ we have that $x_{n} / x_{n-2} \geq 1$, and hence $r_{n}=1$. Similarly, if $r_{n-2}=0$ and $r_{n-1}=1$, then $r_{n}=0$. Hence, if $r_{n_{0}} \neq r_{n_{0}+1}$ for some $n_{0} \geq$ 0 , then the sequences $\left\{r_{n_{0}+2 i}\right\}_{i \geq 0}$ and $\left\{r_{n_{0}+2 i+1}\right\}_{i \geq 0}$ are both constant, as required. This confirms the statement in the theorem, in this case. Otherwise, $\left\{r_{i}\right\}_{i \geq 0}$ itself is a constant sequence and the result again follows.

Remark 2.2. Note that Theorem 2.1 guarantees only the eventually monotonicity of the sequences $\left\{x_{2 n}\right\}$ and $\left\{x_{2 n+1}\right\}$. Hence, for a solution $\left\{x_{n}\right\}$ of (1.1), one of these two subsequences can be infinite. See, for example, [27, Theorem 1], where it was shown that for the case $f(x)=g(x)=x^{p}, p \geq 1,(1.1)$ has unbounded solutions. The problem was previously treated in the papers $[1,11]$ but the proofs appearing there have a gap (for more details see [27]).

The first special case of the nonrational (1.1) was considered in the paper [22], where the second author considered the equation

$$
x_{n}=\frac{\alpha+\beta x_{n-2}}{1+g\left(x_{n-1}\right)}
$$

where the function $g$ satisfies the following conditions:

(i) $g(x)>0$ for $x \geq 0$,

(ii) $g(x)$ is increasing on $[0, \infty)$,

(iii) $x / g(x)$ is increasing on $[0, \infty)$.

The following two conjectures, which were posed by the second author, have circulated among the experts in the field, since early 2001. 
Conjecture 2.3. Assume that $\beta=1$. Show that every solution of (2.3) is bounded.

Conjecture 2.4. Assume that $\beta=1$. Show that every prime periodic solution of (2.3) has period equal to one or two.

Conjecture 2.3 was confirmed in [28] where the following lemma was proved.

Lemma 2.5. Suppose $h$ is a function which satisfies the following conditions:

(a) $h(0) \geq 0$,

(b) $h(x)$ is increasing on $[0, \infty)$,

(c) $x / h(x)$ is nondecreasing on $[0, \infty)$.

Then for given $l, L, \alpha>0$ such that $l<L$, there exist $l_{0}$ and $L_{0}$ such that

(1) $0<l_{0} \leq l$ and $L \leq L_{0}$,

(2) $l_{0} h\left(L_{0}\right) \leq \alpha \leq h\left(l_{0}\right) L_{0}$.

By Lemma 2.5, a result was proven concerning an extension of (2.3). We present the proof of the theorem for the case of (2.3), for the benefit of the reader, since the proof is instructive. For related results regarding boundedness, see, for example, [1, 6, 10, 22, 27, 31, 33, 34].

Theorem 2.6. Assume that $g$ is a function which satisfies conditions (ii) and (iii) and that $g(0) \geq 0$. Consider (2.3) where $\alpha, x_{-2}, x_{-1} \in(0, \infty)$. Then every solution of $(2.3)$ is bounded and persists.

Proof. Choose $l$ and $L$ such that $L>\max \left\{x_{-2}, x_{-1}\right\}$ and $\min \left\{x_{-2}, x_{-1}\right\}>l>0$. By Lemma 2.5, we may also assume that $l g(L) \leq \alpha \leq g(l) L$. Now we may use mathematical induction to prove the result. Assume the statement is true for $x_{-2}, x_{-1}, x_{0}, \ldots, x_{n}$, that is,

$$
l \leq x_{i} \leq L \quad \forall i=-2,-1,0,1, \ldots, n-1 .
$$

Then

$$
x_{n}=\frac{\alpha+x_{n-2}}{1+g\left(x_{n-1}\right)} \leq \frac{\alpha+L}{1+g(l)} .
$$

We claim that $(\alpha+L) /(1+g(l)) \leq L$. But this is obvious since

$$
\alpha+L \leq(1+g(l)) L \Longleftrightarrow \alpha \leq g(l) L .
$$

Similarly, we have that

$$
x_{n}=\frac{\alpha+x_{n-2}}{1+g\left(x_{n-1}\right)} \geq \frac{\alpha+l}{1+g(L)},
$$

from which it follows that $x_{n} \geq l$ for $n=-2,-1,0, \ldots$, completing the proof of the theorem.

By Theorems 2.1 and 2.6 we confirm Conjecture 2.4. Indeed, by Theorem 2.6 we have that every solution $\left\{x_{n}\right\}$ of (2.3) is bounded. On the other hand, by Theorem 2.1, the sequences $\left\{x_{2 n}\right\}$ and $\left\{x_{2 n-1}\right\}$ are eventually monotone, thus convergent. Hence, if (2.3) has periodic solutions they have period one or two, as conjectured. 


\section{Global periodicity of $(1.1)$}

Definition 3.1. Let $g$ be a function defined on a subset of $\mathbb{R}^{n}$. Say that the difference equation

$$
x_{n}=g\left(x_{n-1}, \ldots, x_{n-k}\right), \quad n \in \mathbb{N}_{0}
$$

where $k \in \mathbb{N}$, is periodic, if every solution of (3.1) is periodic.

Periodic equations have been investigated, for example, in $[2,7,8,12,14,16-19,26]$ (see also the references therein).

In this section, we investigate periodic equations of type (1.1). In order to facilitate notation we will write (1.1) in the equivalent form

$$
x_{n}=h\left(x_{n-1}\right) f\left(x_{n-2}\right), \quad n \in \mathbb{N}_{0} .
$$

If every solution of (3.2) is periodic with period $p=2$, then it must hold that

$$
x=h(y) f(x), \quad x, y \in(0, \infty),
$$

that is $x / f(x)=h(y)$, which implies that $f(x)=c x$ and $h(y)=c$ for some positive constant c. Thus, (3.2) has the form $x_{n}=c^{2} x_{n-2}$. Since every solution of (3.2) must be two periodic, it follows that $c=1$. Hence, the equation $x_{n}=x_{n-2}$ is a unique equation of type (3.2) for which all solutions are periodic with period two.

Further we consider those equations of type (3.2) for which all solutions are periodic with period three. For a mapping $f: X \rightarrow X$ the sequence of iterates $\left\{f^{[m]}\right\}_{m \in \mathbb{N} \cup\{0\}}$ of $f$, is defined by $f^{[0]}=I(I$ is the identity function on $X), f^{[1]}=f$ and generally $f^{[m+1]}=f \circ f^{[m]}$ for any $m \in \mathbb{N}$.

Before we prove the result concerning the case, we need the following auxiliary result which is folklore.

Lemma 3.2. Assume that $f:(0, \infty) \rightarrow(0, \infty)$ is a continuous function such that

$$
f^{[6]}(x)=x, \quad x \in(0, \infty) .
$$

Then, $f(x)=x, x \in(0, \infty)$ or $f^{[2]}(x)=x$.

Proof. If $f(x)=f(y)$, then from (3.4) it follows that

$$
x=f^{[6]}(x)=f^{[6]}(y)=y,
$$

which implies that the function $f$ must be $1-1$. Since $f$ is a continuous function we have that $f$ must be strictly monotone.

First assume that $f$ is strictly increasing. If there is a point $x_{0} \in I$ such that $x_{0}<f\left(x_{0}\right)$, then by the monotonicity of $f$ we have

$$
x_{0}<f\left(x_{0}\right)<f^{[2]}\left(x_{0}\right)<\cdots<f^{[6]}\left(x_{0}\right)=x_{0},
$$

which is a contradiction. 
If $x_{0}>f\left(x_{0}\right)$, then we have

$$
x_{0}>f\left(x_{0}\right)>f^{[2]}\left(x_{0}\right)>\cdots>f^{[6]}\left(x_{0}\right)=x_{0},
$$

arriving again at a contradiction.

From this it follows that $f(x)=x$ for every $x \in(0, \infty)$.

Assume now that $f$ is strictly decreasing. Then the function $g(x)=f^{[2]}(x)$ is strictly increasing and

$$
g^{[3]}(x)=\left(f^{[2]}\right)^{[3]}(x)=x .
$$

Similar to the first case, we obtain that $f^{[2]}(x) \equiv x$, finishing the proof of the lemma.

Remark 3.3. Note that if $f^{[2]}(x)=x$, then $f$ is decreasing, maps interval $(0, \infty), " 1-1$," and onto itself, and its graph is symmetric with respect to the line $y=x$, since $f(x)=f^{-1}(x)$.

Theorem 3.4. All solutions of (3.2) are periodic with period three if and only if $f(x)=c_{1} / x$ and $h(x)=c_{2} / x$, for some positive constants $c_{1}$ and $c_{2}$.

Proof. Assume that $f(x)=c_{1} / x$ and $h(x)=c_{2} / x$, for some positive constants $c_{1}$ and $c_{2}$. Then (3.2) becomes

$$
x_{n}=\frac{c_{3}}{x_{n-1} x_{n-2}}, \quad c_{3}=c_{1} c_{2} .
$$

It is easy to see that every solution of the equation is periodic with period three (see, e.g., [12]).

Assume now that every solution of (3.2) is periodic with period three. Then, we have that

$$
u=h(y) f(x), \quad x=h(u) f(y), \quad y=h(x) f(u),
$$

for every $x, y \in(0, \infty)$.

Eliminating $u$ in (3.10) we obtain that

$$
x=h(h(y) f(x)) f(y), \quad y=h(x) f(h(y) f(x)) .
$$

Now, in each of the two equations (3.11), we choose that a variable is arbitrary and the other is equal to 1 , and use the changes

$$
\widehat{h}(x)=h(x) f(1), \quad \widehat{f}(x)=f(x) h(1) .
$$

Then, we obtain

$$
\begin{aligned}
& \widehat{h}(\widehat{f}(z))=z, \quad \widehat{h}(\widehat{h}(z)) \widehat{f}(z)=C, \\
& \widehat{f}(\widehat{f}(z)) \hat{h}(z)=C, \quad \widehat{f}(\widehat{h}(z))=z,
\end{aligned}
$$

for every $z \in(0, \infty)$, where $C=f(1) h(1)$. 
From (3.13) we have

$$
\widehat{f}(\widehat{h}(z))=\widehat{h}(\widehat{f}(z))=z
$$

which implies that

$$
\widehat{f}(z)=\widehat{h}^{-1}(z)
$$

If we set $z \rightarrow \widehat{h}(z)$ in the second identity in (3.13) and $z \rightarrow \widehat{f}(z)$ in the third identity, and then apply (3.14), we obtain

$$
\widehat{f}^{[3]}(z)=\widehat{h}^{[3]}(z)=\frac{C}{z} .
$$

From (3.15) and (3.16) it follows that

$$
\widehat{h}^{[6]}(z)=z \text {. }
$$

Lemma 2.5 implies that $\widehat{h}(z)=z$ or $\widehat{h}^{[2]}(z)=z$. If $\widehat{h}(z)=z$, then (3.13) implies $\widehat{f}(z)=z$, and by the second identity in (3.13) we obtain $z=C$, which is a contradiction.Hence,

$$
\widehat{h}^{[2]}(z)=\widehat{f}^{[2]}(z)=z \text {. }
$$

Substituting (3.18) in (3.13) we obtain that

$$
\widehat{h}(z)=\widehat{f}(z)=\frac{C}{z}
$$

from which it follows that

$$
h(z)=\frac{h(1)}{z}, \quad f(z)=\frac{f(1)}{z}
$$

as desired.

Remark 3.5. It is expected that Theorem 3.4 can be generalized for the case when all solutions of (3.2) are periodic with period more than three.

In the case when all solutions of (3.2) are periodic with period four, the functions $f$ and $g$ must satisfy the following system of functional equations:

$$
\begin{aligned}
& x=h[h(h(y) \cdot f(x)) \cdot f(y)] \cdot f(h(y) f(x)), \\
& y=h(x) \cdot f[h(h(y) \cdot f(x)) \cdot f(y)] .
\end{aligned}
$$

From the system as in the proof of Theorem 3.4, it can be obtained that the functions $\widehat{h}$ and $\widehat{f}$ satisfy the following identities:

$$
\begin{gathered}
\widehat{h}\left(\widehat{h}(\widehat{h}(z)) \widehat{f}(z) C^{-1}\right) \widehat{f}(\widehat{h}(z))=C, \quad \widehat{h}(\widehat{h}(\widehat{f}(z))) \widehat{f}(\widehat{f}(z))=C z, \\
\widehat{h}(z) \widehat{f}(\widehat{h}(\widehat{f}(z)))=C, \quad \widehat{f}\left(\widehat{h}(\widehat{h}(z)) \widehat{f}(z) C^{-1}\right)=z,
\end{gathered}
$$

where $C=f(1) h(1)$.

An obvious solution of the system is $h(x)=c_{1}$ and $f(x)=c_{2} / x$, where $c_{1}, c_{2} \in(0, \infty)$. We leave the problem of finding all solutions of the system as a further direction for investigation for interested readers. 
Note. An early draft by the second author of the paper, containing only results in Section 3, has circulated among the experts since the end of 2005 and was the starting point for further important investigations in the research field, see, for example, $[2,30]$. The paper in the present form is a slight modification of a version from March 2006. A minor publication mishap caused some wrong citations (see [30]), as well as a delay in publishing of it.

\section{Acknowledgments}

The authors are grateful to B. T. Lamb and A. C. Pecorella for useful discussions which led to the current proof of Theorem 2.1.

\section{References}

[1] A. M. Amleh, E. A. Grove, G. Ladas, and D. A. Georgiou, "On the recursive sequence $x_{n+1}=\alpha+$ $x_{n-1} / x_{n}, "$ Journal of Mathematical Analysis and Applications, vol. 233, no. 2, pp. 790-798, 1999.

[2] F. Balibrea, A. Linero Bas, G. S. López, and S. Stević, "Global periodicity of $x_{n+k+1}=$ $f_{k}\left(x_{n+k}\right) \ldots f_{1}\left(x_{n+1}\right)$," Journal of Difference Equations and Applications, vol. 13, no. 10, pp. 901-910, 2007.

[3] K.S. Berenhaut, K. M. Donadio, and J. D. Foley, "On the rational recursive sequence $y_{n}=A+y_{n-1} / y_{n-m}$ for small $A$," to appear in Applied Mathematics Letters.

[4] K. S. Berenhaut, J. E. Dice, J. D. Foley, B. D. Iričanin, and S. Stević, "Periodic solutions of the rational difference equation $y_{n}=y_{n-3}+y_{n-4} / y_{n-1}$," Journal of Difference Equations and Applications, vol. 12, no. 2, pp. 183-189, 2006.

[5] K.S. Berenhaut and S. Stević, "A note on the difference equation $x_{n+1}=1 / x_{n} x_{n-1}+1 / x_{n-3} x_{n-4}$," Journal of Difference Equations and Applications, vol. 11, no. 14, pp. 1225-1228, 2005.

[6] K. S. Berenhaut and S. Stević, "The behaviour of the positive solutions of the difference equation $x_{n}=A+\left(x_{n-2} / x_{n-1}\right)^{p}$," Journal of Difference Equations and Applications, vol. 12, no. 9, pp. 909-918, 2006.

[7] L. Berg, "Nonlinear difference equations with periodic solutions," Rostocker Mathematisches Kolloquium, no. 61, pp. 13-20, 2006.

[8] L. Berg and S. Stević, "Periodicity of some classes of holomorphic difference equations," Journal of Difference Equations and Applications, vol. 12, no. 8, pp. 827-835, 2006.

[9] M. Csörnyei and M. Laczkovich, "Some periodic and non-periodic recursions," Monatshefte für Mathematik, vol. 132, no. 3, pp. 215-236, 2001.

[10] R. DeVault, C. Kent, and W. Kosmala, "On the recursive sequence $x_{n+1}=p+x_{n-k} / x_{n}$," Journal of Difference Equations and Applications, vol. 9, no. 8, pp. 721-730, 2003.

[11] H. M. El-Owaidy, A. M. Ahmed, and M. S. Mousa, "On asymptotic behaviour of the difference equation $x_{n+1}=\alpha+x_{n-1}^{p} / x_{n}^{p}$," Journal of Applied Mathematics $\mathcal{E}$ Computing, vol. 12, no. 1-2, pp. 31-37, 2003.

[12] E. A. Grove and G. Ladas, Periodicities in Nonlinear Difference Equations, vol. 4 of Advances in Discrete Mathematics and Applications, Chapman \& Hall/CRC, Boca Raton, Fla, USA, 2005.

[13] B. D. Iričanin, "A global convergence result for a higher order difference equation," Discrete Dynamics in Nature and Society, vol. 2007, Article ID 91292, 7 pages, 2007.

[14] B. D. Iričanin and S. Stević, "Some systems of nonlinear difference equations of higher order with periodic solutions," Dynamics of Continuous, Discrete E Impulsive Systems. Series A, vol. 13, no. 3-4, pp. 499-507, 2006.

[15] G. Karakostas, "Asymptotic 2-periodic difference equations with diagonally self-invertible responses," Journal of Difference Equations and Applications, vol. 6, no. 3, pp. 329-335, 2000

[16] R. P. Kurshan and B. Gopinath, "Recursively generated periodic sequences," Canadian Journal of Mathematics, vol. 26, pp. 1356-1371, 1974.

[17] R. C. Lyness, "1581. Cycles," The Mathematical Gazette, vol. 26, no. 268, p. 62, 1942.

[18] R. C. Lyness, "1847. Cycles," The Mathematical Gazette, vol. 29, no. 287, pp. 231-233, 1945.

[19] R. C. Lyness, "2952. Cycles," The Mathematical Gazette, vol. 45, no. 353, pp. 207-209, 1961.

[20] S. Stević, "On the recursive sequence $x_{n+1}=x_{n+1} / g\left(x_{n}\right)$," Taiwanese Journal of Mathematics, vol. 6 , no. 3 , pp. 405-414, 2002. 
[21] S. Stević, "Asymptotic behavior of a nonlinear difference equation," Indian Journal of Pure and Applied Mathematics, vol. 34, no. 12, pp. 1681-1687, 2003.

[22] S. Stević, "On the recursive sequence $x_{n+1}=\alpha+\beta x_{n-1} / 1+g\left(x_{n}\right)$," Indian Journal of Pure and Applied Mathematics, vol. 33, no. 12, pp. 1767-1774, 2002.

[23] S. Stević, "On the recursive sequence $x_{n+1}=A / \Pi_{i=0}^{k} x_{n-i}+1 / \Pi_{j=k+2}^{2(k+1)} x_{n-j}$," Taiwanese Journal of Mathematics, vol. 7, no. 2, pp. 249-259, 2003.

[24] S. Stević, "On the recursive sequence $x_{n+1}=\alpha_{n}+x_{n-1} / x_{n}$ II," Dynamics of Continuous, Discrete $\mathcal{E}$ Impulsive Systems. Series A, vol. 10, no. 6, pp. 911-916, 2003.

[25] S. Stević, "A note on periodic character of a difference equation," Journal of Difference Equations and Applications, vol. 10, no. 10, pp. 929-932, 2004.

[26] S. Stević, "Periodic character of a difference equation," Rostocker Mathematisches Kolloquium, no. 59, pp. 3-10, 2005.

[27] S. Stević, "On the recursive sequence $x_{n+1}=\alpha_{n}+x_{n-1}^{p} / x_{n^{\prime}}^{p}$ " Journal of Applied Mathematics $\mathcal{E}$ Computing, vol. 18, no. 1-2, pp. 229-234, 2005.

[28] S. Stević, "On the recursive sequence $x_{n+1}=\alpha+\beta x_{n-k} / f\left(x_{n}, \ldots, x_{n-k+1}\right)$," Taiwanese Journal of Mathematics, vol. 9, no. 4, pp. 583-593, 2005.

[29] S. Stević, "A note on periodic character of a higher order difference equation," Rostocker Mathematisches Kolloquium, no. 61, pp. 21-30, 2006.

[30] S. Stević, "On global periodicity of a class of difference equations," Discrete Dynamics in Nature and Society, vol. 2007, Article ID 23503, 10 pages, 2007.

[31] S. Stević, "On the recursive sequence $x_{n}=\alpha+\sum_{i=1}^{k} \alpha_{i} x_{n-p_{i}} / 1+\sum_{j=1}^{m} \beta_{j} x_{n-q_{j}}$," Journal of Difference Equations and Applications, vol. 13, no. 1, pp. 41-46, 2007.

[32] S. Stević, "On the recursive sequence $x_{n}=1+\sum_{i=1}^{k} \alpha_{i} x_{n-p_{i}} / \sum_{j=1}^{m} \beta_{j} x_{n-q_{j}}$ " Discrete Dynamics in Nature and Society, vol. 2007, Article ID 39404, 7 pages, 2007.

[33] S. Stević, "On the recursive sequence $x_{n+1}=A+x_{n}^{p} / x_{n-1}^{p}$," Discrete Dynamics in Nature and Society, vol. 2007, Article ID 34517, 9 pages, 2007.

[34] T. Sun, H. Xi, and H. Wu, "On boundedness of the solutions of the difference equation $x_{n+1}=x_{n-1} /(p+$ $\left.x_{n}\right)$," Discrete Dynamics in Nature and Society, vol. 2006, Article ID 20652, 7 pages, 2006.

[35] S.-E. Takahasi, Y. Miura, and T. Miura, "On convergence of a recursive sequence $f\left(x_{n-1}, x_{n}\right)$," Taiwanese Journal of Mathematics, vol. 10, no. 3, pp. 631-638, 2006. 


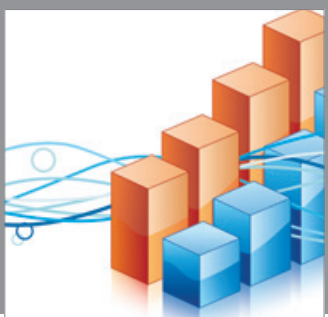

Advances in

Operations Research

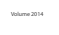

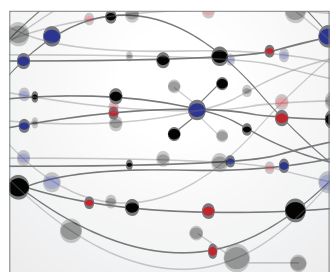

\section{The Scientific} World Journal
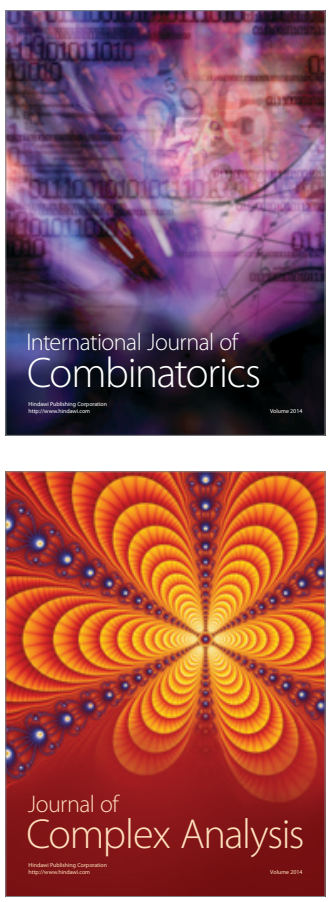

International Journal of

Mathematics and

Mathematical

Sciences
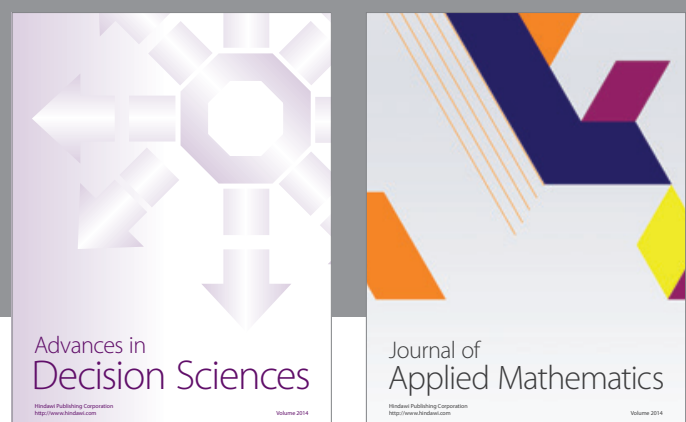

Journal of

Applied Mathematics
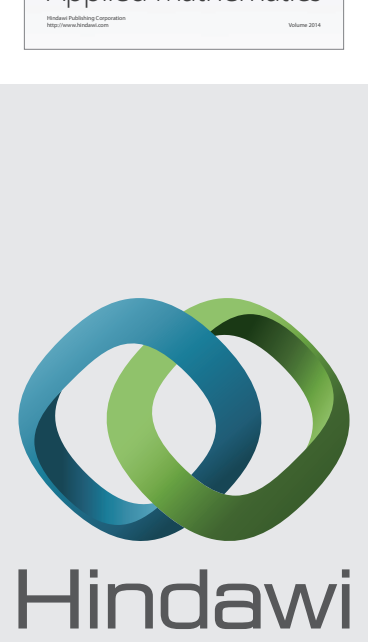

Submit your manuscripts at http://www.hindawi.com
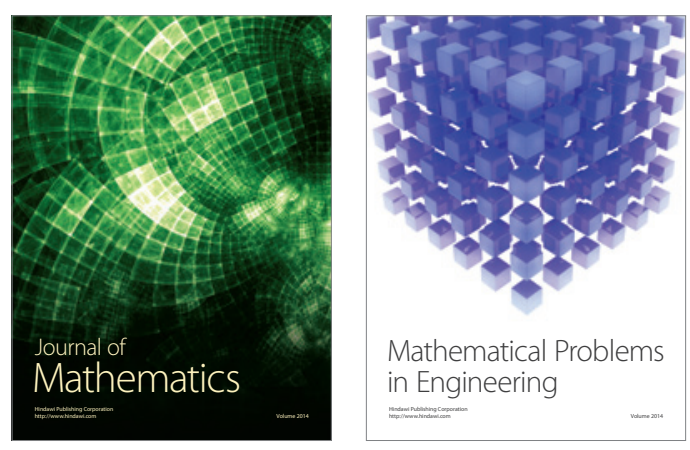

Mathematical Problems in Engineering
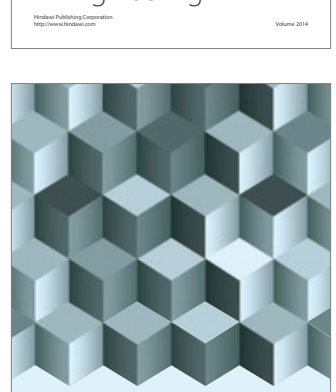

Journal of

Function Spaces
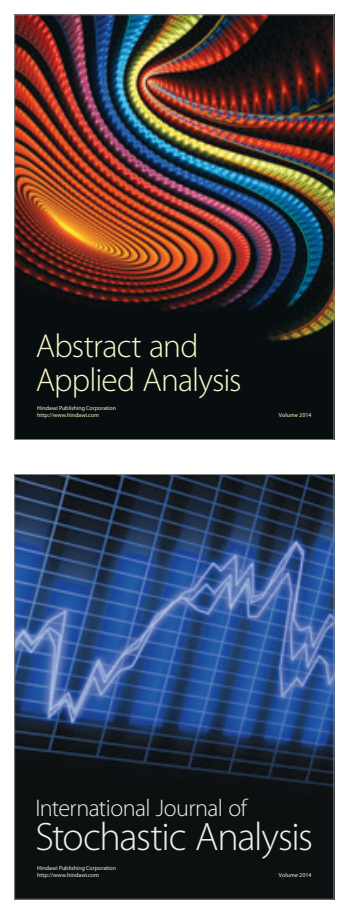

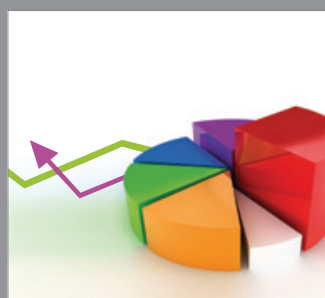

ournal of

Probability and Statistics

Promensencen
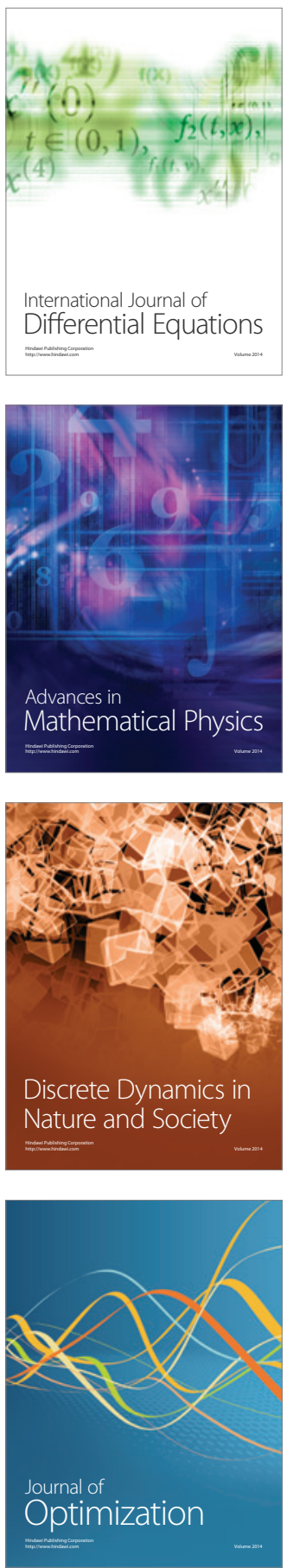